\title{
A terminating evaluation-driven variant of G3i
}

\author{
Mauro Ferrari ${ }^{1}$, Camillo Fiorentini ${ }^{2}$, Guido Fiorino ${ }^{3}$ \\ 1 DiSTA, Univ. degli Studi dell'Insubria, Via Mazzini, 5, 21100, Varese, Italy \\ 2 DI, Univ. degli Studi di Milano, Via Comelico, 39, 20135 Milano, Italy \\ 3 DISCO, Univ. degli Studi di Milano-Bicocca, Viale Sarca, 336, 20126, Milano, Italy
}

\begin{abstract}
We present $\mathbf{G b u}$, a terminating variant of the sequent calculus G3i for intuitionistic propositional logic. Gbu modifies G3i by annotating the sequents so to distinguish rule applications into two phases: an unblocked phase where any rule can be backward applied, and a blocked phase where only right rules can be used. Derivations of $\mathbf{G b u}$ have a trivial translation into G3i. Rules for right implication exploit an evaluation relation, defined on sequents; this is the key tool to avoid the generation of branches of infinite length in proof-search. To prove the completeness of $\mathbf{G b u}$, we introduce a refutation calculus $\mathbf{R b u}$ for unprovability dual to $\mathbf{G b u}$. We provide a proof-search procedure that, given a sequent as input, returns either a $\mathbf{R b u}$-derivation or a $\mathbf{G b u}$-derivation of it.
\end{abstract}

\section{Introduction}

It is well-known that G3i [10], the sequent calculus for intuitionistic propositional logic with weakening and contraction "absorbed" in the rules, is not suited for proof-search. Indeed, the naïve proof-search strategy, consisting in applying the rules of the calculus bottom-up until possible, is not terminating. This is because the rule for left implication retains the main formula $A \rightarrow B$ in the left-hand side premise, hence such a formula might be selected for application more and more times. A possible solution to this problem is to support the proofsearch procedure with a loop-checking mechanism [5-7]: whenever the "same" sequent occurs twice along a branch of the proof under construction, the search is cut. An efficient implementation of loop-checking exploits histories [6,7]. In the construction of a branch, the formulas decomposed by right rules are stored in the history; loops are avoided by preventing the application of some right rules to formulas in the history.

In this paper we propose a different and original approach: we show that terminating proof-search for G3i can be accomplished only exploiting the information contained in the sequent to be proved by means of a suitable evaluation relation. Our proof-search strategy alternates two phases: an unblocked phase (u-phase), where all the rules of G3i can be backward applied, and a blocked phase (b-phase), where only right-rules can be used. To improve the presentation, we embed the strategy inside the calculus by annotating sequents with the label u (unblocked) or b (blocked); we call Gbu the resulting calculus (see Fig. 1). A Gbu-derivation can be straightforwardly mapped to a G3i-derivation 
by erasing the labels and, possibly, by padding the left contexts; from this, the soundness of Gbu immediately follows. Unblocked sequents, characterizing an u-phase, behave as the ordinary sequents of G3i: any rule of Gbu can be (backward) applied to them. Instead, b-sequents resemble focused-right sequents (see, e.g., [2]): they only allow backward right-rule applications (thus, the left context is "blocked"). Proof-search starts from an u-sequent (u-phase); the transition to a b-phase is determined by the application of one of the rules for left implication or right disjunction. For instance, let $[A \rightarrow B, \Gamma \stackrel{\mathrm{u}}{\Rightarrow} H]$ be the u-sequent to be proved and suppose we apply the rule $\rightarrow L$ with main formula $A \rightarrow B$. The next goals are the b-sequent $[A \rightarrow B, \Gamma \stackrel{\mathrm{b}}{\Rightarrow} A]$ and the u-sequent $[B, \Gamma \stackrel{\mathrm{u}}{\Rightarrow} H]$, corresponding to the two premises of $\rightarrow L$. While the latter goal continues the u-phase, the former one starts a new b-phase, which focuses on $A$. Similarly, if we apply the rule $\vee R_{k}$ (with $k \in\{0,1\}$ ) to $\left[\Gamma \stackrel{\mathrm{u}}{\Rightarrow} H_{0} \vee H_{1}\right]$, the phase changes to $\mathrm{b}$ and the next goal is $\left[\Gamma \stackrel{\mathrm{b}}{\Rightarrow} H_{k}\right]$, the only premise of $\vee R_{k}$.

Rules for right implication have two possible outcomes determined by the evaluation relation. Indeed, let $[\Gamma \stackrel{l}{\Rightarrow} A \rightarrow B]$ be the current goal $(l \in\{\mathrm{u}, \mathrm{b}\})$ and let $A \rightarrow B$ be the selected main formula: if $A$ is evaluated in $\Gamma$, then we continue the search with $[\Gamma \stackrel{l}{\Rightarrow} B]$ and the phase does not change (see rule $\rightarrow R_{1}$ ); note that the formula $A$ is dropped out. If $A$ is not evaluated in $\Gamma$ the next goal is $[A, \Gamma \stackrel{\mathrm{u}}{\Rightarrow} B]$. Moreover, if $l=\mathrm{b}$, we switch from a b-phase to an u-phase and this is the only case where a b-sequent is "unblocked". The crucial point is that, due to the side conditions on the application of rules $\rightarrow R_{1}$ and $\rightarrow R_{2}$ (which rely on the evaluation relation), every branch of a Gbu-tree has finite length (Section 3); this implies that our proof-search strategy always terminates. We point out that we do not bound ourselves to a specific evaluation relation, but we admit any evaluation relation satisfying properties $(\mathcal{E} 1)-(\mathcal{E} 6)$ defined in Section 2 .

The proof of completeness ( $[\Gamma \Rightarrow H]$ provable in G3i implies $[\Gamma \stackrel{\mathrm{u}}{\Rightarrow} H]$ provable in $\mathbf{G b u}$ ) involves non-trivial aspects. Following [3, 9], we introduce a refutation calculus $\mathbf{R b u}$ for asserting intuitionistic unprovability (Section 4). From an Rbu-derivation of an u-sequent $\sigma^{\mathrm{u}}=[\Gamma \stackrel{\mathrm{u}}{\Rightarrow} H]$ we can extract a Kripke countermodel for $\sigma^{\mathrm{u}}$, namely a Kripke model such that, at its root, all formulas in $\Gamma$ are forced and $H$ is not forced; from this, it follows that $\sigma^{\mathrm{u}}$ is not intuitionistically valid. In Section 5 we introduce the function $F$ which implements the proofsearch strategy outlined above; if the search for a Gbu-derivation of $\sigma^{\mathrm{u}}$ fails, an Rbu-derivation of $\sigma^{\mathrm{u}}$ is built. To sum up, $\mathrm{F}\left(\sigma^{\mathrm{u}}\right)$ returns either a $\mathbf{G b u}$-derivation or an Rbu-derivation of $\sigma^{\mathrm{u}}$; in the former case we get a G3i-derivation of the sequent $\sigma=[\Gamma \Rightarrow H]$, in the latter case we can build a countermodel for $\sigma$.

\section{Preliminaries and evaluations}

We consider the propositional language $\mathcal{L}$ based on a denumerable set of propositional variables $\mathcal{V}$, the connectives $\wedge, \vee, \rightarrow$ and the logical constant $\perp$. We denote with $\mathcal{V}(A)$ the set of propositional variables occurring in $A$, with $|A|$ the size of $A$, that is the number of symbols occurring in $A$, and with $\operatorname{Sf}(A)$ the set of subformulas of $A$ (including $A$ itself). 
A (finite) Kripke model for $\mathcal{L}$ is a structure $\mathcal{K}=\langle P, \leq, \rho, V\rangle$, where $\langle P, \leq, \rho\rangle$ is a finite partially ordered set with minimum $\rho$ and $V: P \rightarrow 2^{\mathcal{V}}$ is a function such that $\alpha \leq \beta$ implies $V(\alpha) \subseteq V(\beta)$. The forcing relation $\Vdash \subseteq P \times \mathcal{L}$ is defined as follows:

$-\mathcal{K}, \alpha \nVdash \perp$ and, for every $p \in \mathcal{V}, \mathcal{K}, \alpha \Vdash p$ iff $p \in V(\alpha)$;

$-\mathcal{K}, \alpha \Vdash A \wedge B$ iff $\mathcal{K}, \alpha \Vdash A$ and $\mathcal{K}, \alpha \Vdash B$;

$-\mathcal{K}, \alpha \Vdash A \vee B$ iff $\mathcal{K}, \alpha \Vdash A$ or $\mathcal{K}, \alpha \Vdash B$;

$-\mathcal{K}, \alpha \Vdash A \rightarrow B$ iff, for every $\beta \in P$ such that $\alpha \leq \beta, \mathcal{K}, \beta \nVdash A$ or $\mathcal{K}, \beta \Vdash B$.

Given a set $\Gamma$ of formulas, $\mathcal{K}, \alpha \Vdash \Gamma$ iff $\mathcal{K}, \alpha \Vdash A$ for every $A \in \Gamma$. Monotonicity property holds for arbitrary formulas, i.e.: $\mathcal{K}, \alpha \Vdash A$ and $\alpha \leq \beta$ imply $\mathcal{K}, \beta \Vdash A$. A formula $A$ is valid in $\mathcal{K}$ iff $\mathcal{K}, \rho \Vdash A$. Intuitionistic propositional logic coincides with the set of the formulas valid in all (finite) Kripke models [1].

As motivated in the Introduction, we use (labelled) sequents of the form $\sigma=[\Gamma \stackrel{l}{\Rightarrow} H]$ where $l \in\{\mathrm{b}, \mathrm{u}\}, \Gamma$ is a finite set of formulas and $H$ is a formula. We adopt the usual notational conventions; e.g., $[A, \Gamma \stackrel{l}{\Rightarrow} H]$ stands for $[\{A\} \cup \Gamma \stackrel{l}{\Rightarrow} H]$. The size of $\sigma$ is $|\sigma|=\sum_{A \in \Gamma}|A|+|H|$; the set of subformulas of $\sigma$ is $\operatorname{Sf}(\sigma)=$ $\bigcup_{A \in \Gamma \cup\{H\}} \operatorname{Sf}(A)$.

The semantics of formulas extends to sequents as follows. Given a Kripke model $\mathcal{K}$ and a world $\alpha$ of $\mathcal{K}, \alpha$ refutes $\sigma=[\Gamma \stackrel{l}{\Rightarrow} H]$ in $\mathcal{K}$, written $\mathcal{K}, \alpha \triangleright \sigma$, iff $\mathcal{K}, \alpha \Vdash \Gamma$ and $\mathcal{K}, \alpha \nVdash H ; \sigma$ is refutable if there exists a Kripke model $\mathcal{K}$ with root $\rho$ such that $\mathcal{K}, \rho \triangleright \sigma$; in this case $\mathcal{K}$ is a countermodel for $\sigma$. It is easy to check that $\sigma$ is refutable iff the formula $\wedge \Gamma \rightarrow H$ is not intuitionistically valid iff, by soundness and completeness of G3i $[10],[\Gamma \Rightarrow H]$ is not provable in G3i.

Evaluations An evaluation relation $\vdash_{\mathcal{E}}$ is a relation between a set $\Gamma$ of formulas and a formula $A$ satisfying the following properties:

$(\mathcal{E} 1) \Gamma \vdash_{\mathcal{E}} A$ iff $\Gamma \cap \operatorname{Sf}(A) \vdash_{\mathcal{E}} A$.

$(\mathcal{E} 2) A, \Gamma \vdash_{\mathcal{E}} A$.

$(\mathcal{E} 3) \Gamma \vdash_{\mathcal{E}} A$ and $\Gamma \vdash_{\mathcal{E}} B$ implies $\Gamma \vdash_{\mathcal{E}} A \wedge B$.

$(\mathcal{E} 4) \Gamma \vdash_{\mathcal{E}} A_{k}$, with $k \in\{0,1\}$, implies $\Gamma \vdash_{\mathcal{E}} A_{0} \vee A_{1}$.

$(\mathcal{E} 5) \Gamma \vdash_{\mathcal{E}} B$ implies $\Gamma \vdash_{\mathcal{E}} A \rightarrow B$.

$(\mathcal{E} 6)$ Let $\mathcal{K}=\langle P, \leq, \rho, V\rangle$ and $\alpha \in P$; if $\mathcal{K}, \alpha \Vdash \Gamma$ and $\Gamma \vdash_{\mathcal{E}} A$, then $\mathcal{K}, \alpha \Vdash A$.

Conditions $(\mathcal{E} 1)-(\mathcal{E} 5)$ concern syntactical properties; note that, by $(\mathcal{E} 1)$, the evaluation of $A$ w.r.t. $\Gamma$ only depends on the subformulas in $\Gamma$ which are subformulas of $A$. Intuitively, the role of an evaluation relation is to check if the "information contained" in $A$ is semantically implied by $\Gamma$ (see $(\mathcal{E} 6)$ ). In the sequel, we also write $[\Gamma \stackrel{l}{\Rightarrow} H] \vdash_{\mathcal{E}} A$ to mean $\Gamma \vdash_{\mathcal{E}} A$.

In the examples we use the evaluation relation $\vdash_{\tilde{\mathcal{E}}}$ defined below. Let $\mathcal{L}_{\top}$ be the language extending $\mathcal{L}$ with the constant $T(\mathcal{K}, \alpha \Vdash \top$, for every $\mathcal{K}$ and every $\alpha$ in $\mathcal{K})$. To define $\vdash_{\tilde{\mathcal{E}}}$, we introduce the function $\mathcal{R}$ which simplifies a formula $A \in \mathcal{L}_{\top}$ w.r.t. a set $\Gamma$ of formulas of $\mathcal{L}$ (see [4]): 


$$
\mathcal{R}(A, \Gamma)= \begin{cases}\top & A \in \Gamma \\ A & \text { if } A \notin \Gamma \text { and } A \in \mathcal{V} \cup\{\perp, \top\} \\ \mathcal{B}\left(\mathcal{R}\left(A_{0}, \Gamma\right) \cdot \mathcal{R}\left(A_{1}, \Gamma\right)\right) & \text { if } A \notin \Gamma \text { and } A=A_{0} \cdot A_{1} \text { with } \cdot \in\{\wedge, \vee, \rightarrow\}\end{cases}
$$

$\mathcal{B}(A)$ performs the boolean simplification of $A[4,8]$, consisting in applying the following reductions inside $A$ :

$$
\begin{array}{llllll}
K \wedge \top \rightsquigarrow K & K \wedge \perp \rightsquigarrow \perp & K \vee \top \rightsquigarrow \top & K \vee \perp \rightsquigarrow K & K \rightarrow \top \rightsquigarrow \top & K \rightarrow K \rightsquigarrow \top \\
\top \wedge K \rightsquigarrow K & \perp \wedge K \rightsquigarrow \perp & \top \vee K \rightsquigarrow \top & \perp \vee K \rightsquigarrow K & \top \rightarrow K \rightsquigarrow K & \perp \rightarrow K \rightsquigarrow \top
\end{array}
$$

We set $\Gamma \vdash_{\tilde{\mathcal{E}}} A$ iff $\mathcal{R}(A, \Gamma)=\top$.

Theorem 1. $\vdash_{\tilde{\mathcal{E}}}$ is an evaluation relation.

Proof. We have to prove that $\vdash_{\tilde{\mathcal{E}}}$ satisfies properties $(\mathcal{E} 1)-(\mathcal{E} 6)$ of Section 2 .

- $(\mathcal{E} 1)$ It is easy to prove, by induction on the structure of $A$, that $\mathcal{R}(A, \Gamma)=$ $\mathcal{R}(A, \Gamma \cap \operatorname{Sf}(A))$, thus $\Gamma \vdash_{\tilde{\mathcal{E}}} A$ iff $\Gamma \cap \operatorname{Sf}(A) \vdash_{\tilde{\mathcal{E}}} A$.

- $(\mathcal{E} 2)$ It immediately follows by the definition of $\vdash_{\tilde{\mathcal{E}}}$ and $\mathcal{R}$.

- (E) Let $\Gamma \vdash_{\tilde{\mathcal{E}}} A$ and $\Gamma \vdash_{\tilde{\mathcal{E}}} B$. By definition of $\vdash_{\tilde{\mathcal{E}}}, \mathcal{R}(A, \Gamma)=\mathcal{R}(B, \Gamma)=\top$. To prove $\Gamma \vdash_{\tilde{\mathcal{E}}} A \wedge B$, we must show that $\mathcal{R}(A \wedge B, \Gamma)=\top$. If $A \wedge B \in \Gamma$, this immediately follows. Otherwise: $\mathcal{R}(A \wedge B, \Gamma)=\mathcal{B}(\mathcal{R}(A, \Gamma) \wedge \mathcal{R}(B, \Gamma))=$ $\mathcal{B}(\top \wedge \top)=\top$. The proof of properties $(\mathcal{E} 4)$ and $(\mathcal{E} 5)$ is similar.

- $(\mathcal{E} 6)$ Let $\mathcal{K}=\langle P, \leq, \rho, V\rangle$ and $\alpha \in P$ such that $\mathcal{K}, \alpha \Vdash \Gamma$. It is easy to prove, by induction on $A$, that $\mathcal{K}, \alpha \Vdash A \leftrightarrow \mathcal{R}(A, \Gamma)$. Now, if $\Gamma \vdash_{\tilde{\mathcal{E}}} A$ then $\mathcal{R}(A, \Gamma)=\mathrm{\top}$; hence by the above property $\mathcal{K}, \alpha \Vdash A \leftrightarrow \top$ and this implies $\mathcal{K}, \alpha \Vdash A$.

\section{The sequent calculus Gbu}

We present the G3-style [10] calculus Gbu for intuitionistic propositional logic. The calculus consists of the axiom rules (rules with zero premises) $\perp L$ and Id, and the left and right introduction rules in Fig. 1. The main formula of a rule is the one put in evidence in the conclusion of the rule. In the conclusion of a rule, when we write $C, \Gamma$ we assume that $C \notin \Gamma$; e.g., in the rule $\wedge L$ it is assumed that $A \wedge B \notin \Gamma$, hence the formula $A \wedge B$ is not retained in the premise. The choice between $\rightarrow R_{1}$ and $\rightarrow R_{2}$ depends on the relation $\vdash_{\mathcal{E}}$. In the application of $\rightarrow L$ to $\sigma=[A \rightarrow B, \Gamma \stackrel{\mathrm{u}}{\Rightarrow} H]$, contraction of $A \rightarrow B$ is explicitly introduced in the leftmost premise $\sigma_{A}$; as a consequence we might have $\left|\sigma_{A}\right| \geq|\sigma|$. In all the other cases, passing from the conclusion to a premise of a rule, the size of the sequents strictly decreases. The rule $\rightarrow R_{2}$ is the only rule that, when applied backward, can turn a b-sequent into an u-sequent.

A Gbu-tree $\pi$ is a tree of sequents such that: if $\sigma$ is a node of $\pi$ with $\sigma_{1}, \ldots, \sigma_{n}$ as children, then there exists a rule of $\mathbf{G b u}$ having premises $\sigma_{1}, \ldots, \sigma_{n}$ and conclusion $\sigma$. The root rule of $\pi$ is the one having as conclusion the root sequent 


$$
\begin{aligned}
& \overline{[\perp, \Gamma \stackrel{l}{\Rightarrow} H]} \perp L \quad \overline{[H, \Gamma \stackrel{l}{\Rightarrow} H]} \mathrm{Id} \\
& \frac{[A, B, \Gamma \stackrel{\mathrm{u}}{\Rightarrow} H]}{[A \wedge B, \Gamma \stackrel{\mathrm{u}}{\Rightarrow} H]} \wedge L \quad \frac{[\Gamma \stackrel{l}{\Rightarrow} A] \quad[\Gamma \stackrel{l}{\Rightarrow} B]}{[\Gamma \stackrel{l}{\Rightarrow} A \wedge B]} \wedge R \\
& \frac{[A, \Gamma \stackrel{\mathrm{u}}{\Rightarrow} H] \quad[B, \Gamma \stackrel{\mathrm{u}}{\Rightarrow} H]}{[A \vee B, \Gamma \stackrel{\mathrm{u}}{\Rightarrow} H]} \vee L \quad \quad \frac{\left[\Gamma \stackrel{\mathrm{b}}{\Rightarrow} H_{k}\right]}{\left[\Gamma \stackrel{\mathrm{l}}{\Rightarrow} H_{0} \vee H_{1}\right]} \vee R_{k} \quad k \in\{0,1\} \\
& \frac{[A \rightarrow B, \Gamma \stackrel{\mathrm{b}}{\Rightarrow} A][B, \Gamma \stackrel{\mathrm{u}}{\Rightarrow} H]}{[A \rightarrow B, \Gamma \stackrel{\mathrm{u}}{\Rightarrow} H]} \rightarrow L \quad \frac{[\Gamma \stackrel{l}{\Rightarrow} B]}{[\Gamma \stackrel{l}{\Rightarrow} A \rightarrow B]} \rightarrow R_{1} \quad \frac{[A, \Gamma \stackrel{\mathrm{u}}{\Rightarrow} B]}{[\Gamma \stackrel{l}{\Rightarrow} A \rightarrow B]} \rightarrow R_{2} \\
& \text { if } \Gamma \vdash_{\mathcal{E}} A \quad \text { if } \Gamma \forall \mathcal{E} A
\end{aligned}
$$

Fig. 1. The calculus Gbu.

of $\pi$. A Gbu-derivation of $\sigma$ is a Gbu-tree $\pi$ with root $\sigma$ and having conclusions of an axiom rule as leaves. A sequent $\sigma$ is provable in $\mathbf{G b u}$ iff there exists a $\mathbf{G b u}$ derivation of $\sigma ; H$ is provable in $\mathbf{G b u}$ iff $[\stackrel{\mathrm{u}}{\Rightarrow} H]$ is provable in $\mathbf{G b u}$. Note that Gbu has the subformula property: given a Gbu-tree $\pi$ with root $\sigma$, for every sequent $\sigma^{\prime}$ occurring in $\pi$ it holds that $\operatorname{Sf}\left(\sigma^{\prime}\right) \subseteq \operatorname{Sf}(\sigma)$.

A Gbu-derivation $\pi$ can be translated into a G3i-derivation $\tilde{\pi}$ applying the following steps: erase the labels from the sequents in $\pi$; when rule $\rightarrow R_{1}$ is applied, add the formula $A$ to the left context; rename all occurrences of $\rightarrow R_{1}$ and $\rightarrow R_{2}$ to $\rightarrow R$. From this translation and the soundness of G3i [10] we get the soundness of $\mathbf{G b u}$. Semantically, this means that, if $\sigma$ is provable in $\mathbf{G b u}$, then $\sigma$ is not refutable.

Here we provide an example of a $\mathbf{G b u}$-derivation, then we prove that $\mathbf{G b u}$ is terminating. The completeness of $\mathbf{G b u}$ (Theorem 4) is proved in Section 5 as a consequence of the correctness of the proof-search procedure.

Example 1. Let $W=((((p \rightarrow q) \rightarrow p) \rightarrow p) \rightarrow q) \rightarrow q$ be an instance of the Weak Pierce Law [1]. In Fig. 2 we give a Gbu-derivation ${ }^{1} \pi_{1}$ of $\sigma_{1}=[\stackrel{u}{\Rightarrow} W]$, using the evaluation $\vdash_{\tilde{\mathcal{E}}}$ of Section 2. Sequents are indexed by integers; by $\pi_{i}$ we denote the subderivation of $\pi_{1}$ with root $\sigma_{i}$. When ambiguities can arise, we underline the main formula of a rule application. Building the derivation bottom-up, the only choice points are in the (backward) application of rule $\rightarrow L$ to $\sigma_{4}$ and $\sigma_{7}$, since we can select both $A$ and $B$ as main formula. If at sequent $\sigma_{6}$ we choose $B$ instead of $A$, we get the $\mathbf{G b u}$-tree with root $\sigma_{6}$ sketched on the right. We have $\sigma_{7^{\prime}} \vdash_{\tilde{\mathcal{E}}} p$ (indeed, $p$ occurs on the left in $\sigma_{7^{\prime}}$ ), hence the rule $\rightarrow R_{1}$ must be applied to $\sigma_{7^{\prime}}$, which

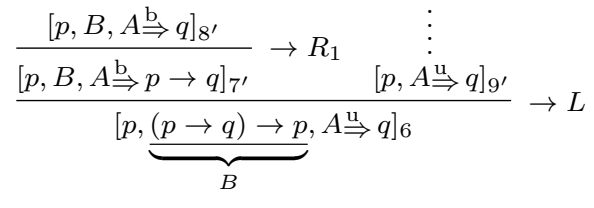

${ }^{1}$ The derivations and their $\mathrm{LTT}_{\mathrm{E}} \mathrm{X}$ rendering are generated with g3ibu, an implementation of $\mathbf{G b u}$ and $\mathbf{R b u}$ available at http://www.dista.uninsubria.it/ ferram/. 


$$
\begin{aligned}
& W=A \rightarrow q \quad A=(B \rightarrow p) \rightarrow q \quad B=(p \rightarrow q) \rightarrow p \\
& \overline{[p, B, A \stackrel{\mathrm{b}}{\Rightarrow} p]_{8}} \text { Id }
\end{aligned}
$$

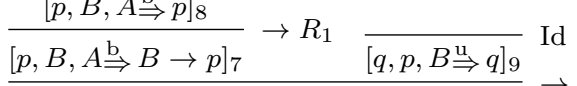

$$
\begin{aligned}
& \frac{[p, B, \underline{A} \stackrel{\text { u }}{\Rightarrow} q]_{6}}{\underline{[B, A \stackrel{\mathrm{b}}{\Rightarrow} p \rightarrow q]_{5}} \rightarrow R_{2} \quad} \rightarrow L \\
& \frac{\frac{[\underline{B}, A \stackrel{\mathrm{u}}{\Rightarrow} p]_{4}}{[A \stackrel{\mathrm{b}}{\Rightarrow} \underbrace{((p \rightarrow q) \rightarrow p) \rightarrow p]_{3}}_{B} \rightarrow R_{2}} \rightarrow L}{\frac{[A \stackrel{\mathrm{u}}{\Rightarrow} q]_{2}}{[q \stackrel{\mathrm{u}}{\Rightarrow} q]_{11}}} \text { Id }
\end{aligned}
$$

Fig. 2. Gbu-derivation of Weak Pierce Law

yields the b-sequent $\sigma_{8^{\prime}}$. Since $\sigma_{8^{\prime}}$ is blocked, we cannot decompose again left implications; thus the proof-search fails without entering an infinite loop.

Termination of Gbu We show that every Gbu-tree has finite depth. A Gbubranch is a sequence of sequents $\mathcal{B}=\left(\sigma_{1}, \sigma_{2}, \ldots\right)$ such that, for every $i \geq 1$, there exists a rule $\mathcal{R}$ of $\mathbf{G b u}$ having $\sigma_{i}$ as conclusion and $\sigma_{i+1}$ among its premises. The length of $\mathcal{B}$ is the number of sequents in it. Let $\gamma=\left(\sigma_{i}, \sigma_{i+1}\right)$ be a pair of successive sequents in $\mathcal{B}$ with labels $l_{i}$ and $l_{i+1}$ respectively; $\gamma$ is a bu-pair if $l_{i}=\mathrm{b}$ and $l_{i+1}=\mathrm{u} ; \gamma$ is an ub-pair if $l_{i}=\mathrm{u}$ and $l_{i+1}=\mathrm{b}$. $\operatorname{By} \operatorname{BU}(\mathcal{B})$ and $\operatorname{UB}(\mathcal{B})$ we denote the number of bu-pairs and ub-pairs occurring in $\mathcal{B}$ respectively. Note that the only rule generating bu-pairs is $\rightarrow R_{2}$. Moreover, $\left|\sigma_{i+1}\right| \geq\left|\sigma_{i}\right|$ can happen only if $\left(\sigma_{i}, \sigma_{i+1}\right)$ is an ub-pair generated by $\rightarrow L: \sigma_{i+1}$ is the leftmost premise of an application of $\rightarrow L$ with conclusion $\sigma_{i}$. As a consequence, every subbranch of $\mathcal{B}$ not containing ub-pairs is finite. Hence, if we show that $\operatorname{UB}(\mathcal{B})$ is finite, we get that $\mathcal{B}$ has finite length.

We prove a kind of persistence of $\vdash_{\mathcal{E}}$, namely: if $A$ occurs in the left-hand side of a sequent $\sigma$ occurring in $\mathcal{B}$, then $\sigma^{\prime} \vdash_{\mathcal{E}} A$ for every $\sigma^{\prime}$ following $\sigma$ in $\mathcal{B}$.

Lemma 1. Let $\mathcal{B}=\left(\sigma_{1}, \sigma_{2}, \ldots\right)$ be a $\mathbf{G b u - b r a n c h ~ w h e r e , ~ f o r ~ e v e r y ~} i \geq 1, \sigma_{i}=$ $\left[\Gamma_{i} \stackrel{l_{i}}{\Rightarrow} H_{i}\right]$. Let $n \geq 1$ and $A \in \bigcup_{1 \leq i \leq n} \Gamma_{i}$. Then, $\Gamma_{n} \vdash_{\mathcal{E}} A$.

Proof. By induction on $|A|$. If $A \in \Gamma_{n}$, by $(\mathcal{E} 2)$ we immediately get $\Gamma_{n} \vdash_{\mathcal{E}} A$. If $A \notin \Gamma_{n}$, there exists $i: 1 \leq i<n$ such that $A \in \Gamma_{i}$ and $A \notin \Gamma_{i+1}$. This implies $A=B \cdot C$ with $\cdot \in\{\wedge, \vee, \rightarrow\}$. Let $\cdot=\wedge$; then $\sigma_{i+1}$ is obtained from $\sigma_{i}$ by an application of $\wedge L$ with main formula $B \wedge C$, hence $B \in \Gamma_{i+1}$ and $C \in \Gamma_{i+1}$. By induction hypothesis, $\Gamma_{n} \vdash_{\mathcal{E}} B$ and $\Gamma_{n} \vdash_{\mathcal{E}} C$; by $(\mathcal{E} 3), \Gamma_{n} \vdash_{\mathcal{E}} B \wedge C$. The cases $\cdot \in\{\vee, \rightarrow\}$ are similar and require properties $(\mathcal{E} 4)$ and $(\mathcal{E} 5)$.

Now, we provide a bound on $\mathrm{BU}(\mathcal{B})$. 


$$
\begin{aligned}
& \overline{\left[\Gamma^{\rightarrow}, \Gamma^{\mathrm{At}} \stackrel{l}{\Rightarrow} H\right]} \quad \text { Irr } \quad \text { if }\left[\Gamma^{\rightarrow}, \Gamma^{\mathrm{At}} \stackrel{l}{\Rightarrow} H\right] \text { is irreducible }\left\{\begin{array}{l}
H=\perp \text { or } H \in \mathcal{V} \backslash \Gamma^{\mathrm{At}} \\
l=\mathrm{b} \text { or } \Gamma^{\rightarrow}=\emptyset
\end{array}\right. \\
& \frac{[A, B, \Gamma \stackrel{\mathrm{u}}{\Rightarrow} H]}{[A \wedge B, \Gamma \stackrel{\mathrm{u}}{\Rightarrow} H]} \wedge L \quad \quad \frac{\left[\Gamma \stackrel{l}{\Rightarrow} H_{k}\right]}{\left[\Gamma \stackrel{l}{\Rightarrow} H_{0} \wedge H_{1}\right]} \wedge R_{k} \quad k \in\{0,1\} \\
& \frac{\left[A_{k}, \Gamma \stackrel{\mathrm{u}}{\Rightarrow} H\right]}{\left[A_{0} \vee A_{1}, \Gamma \stackrel{\mathrm{u}}{\Rightarrow} H\right]} \vee L_{k} \quad k \in\{0,1\} \quad \frac{\left[\Gamma \stackrel{\mathrm{b}}{\Rightarrow} H_{0}\right] \quad\left[\Gamma \stackrel{\mathrm{b}}{\Rightarrow} H_{1}\right]}{\left[\Gamma \stackrel{\mathrm{b}}{\Rightarrow} H_{0} \vee H_{1}\right]} \vee R \\
& \frac{[B, \Gamma \stackrel{\mathrm{u}}{\Rightarrow} H]}{[A \rightarrow B, \Gamma \stackrel{\mathrm{u}}{\Rightarrow} H]} \rightarrow L \quad \begin{array}{rr}
\frac{[\Gamma \stackrel{l}{\Rightarrow} B]}{[\Gamma \stackrel{l}{\Rightarrow} A \rightarrow B]} \rightarrow R_{1} & \frac{[A, \Gamma \stackrel{\mathrm{u}}{\Rightarrow} B]}{[\Gamma \stackrel{l}{\Rightarrow} A \rightarrow B]} \rightarrow R_{2} \\
\text { if } \Gamma \vdash_{\mathcal{E}} A & \text { if } \Gamma \nvdash_{\mathcal{E}} A
\end{array} \\
& \frac{\left\{\left[\Gamma^{\rightarrow}, \Gamma^{\mathrm{At}} \stackrel{\mathrm{b}}{\Rightarrow} A\right]\right\}_{A \rightarrow B \in \Gamma^{\prime} \rightarrow}}{\left[\Gamma^{\rightarrow}, \Gamma^{\mathrm{At}} \stackrel{\mathrm{u}}{\Rightarrow} H\right]} \mathrm{S}_{u}^{\mathrm{At}} \quad \text { where } \Gamma^{\rightarrow} \neq \emptyset \text { and }\left(H=\perp \text { or } H \in \mathcal{V} \backslash \Gamma^{\mathrm{At}}\right) \\
& \frac{\left\{\left[\Gamma^{\rightarrow}, \Gamma^{\mathrm{At}} \stackrel{\mathrm{b}}{\Rightarrow} A\right]\right\}_{A \rightarrow B \in \Gamma^{\prime} \rightarrow}\left[\Gamma^{\rightarrow}, \Gamma^{\mathrm{At}} \stackrel{\mathrm{b}}{\Rightarrow} H_{0}\right] \quad\left[\Gamma^{\rightarrow}, \Gamma^{\mathrm{At}} \stackrel{\mathrm{b}}{\Rightarrow} H_{1}\right]}{\left[\Gamma^{\rightarrow}, \Gamma^{\mathrm{At}} \stackrel{\mathrm{u}}{\Rightarrow} H_{0} \vee H_{1}\right]} \mathrm{S}_{u}^{\vee}
\end{aligned}
$$

Fig. 3. The refutation calculus Rbu.

Lemma 2. Let $\mathcal{B}=\left(\sigma_{1}, \sigma_{2}, \ldots\right)$ be a Gbu-branch. Then, $\mathrm{BU}(\mathcal{B}) \leq\left|\sigma_{1}\right|$.

Proof. Let $\left(\sigma_{i}^{\mathrm{b}}, \sigma_{i+1}^{\mathrm{u}}\right)$ be a bu-pair in $\mathcal{B}$. Since bu-pairs are generated by applications of $\rightarrow R_{2}$, we have: $\sigma_{i}^{\mathrm{b}}=[\Gamma \stackrel{\mathrm{b}}{\Rightarrow} A \rightarrow B], \sigma_{i+1}^{\mathrm{u}}=[A, \Gamma \stackrel{\mathrm{u}}{\Rightarrow} B]$ and $\Gamma \forall_{\mathcal{E}} A$. By Lemma 1 , for every $j \geq i+1$ it holds that $\Gamma_{j} \vdash_{\mathcal{E}} A$. Thus, any bu-pair following $\left(\sigma_{i}^{\mathrm{b}}, \sigma_{i+1}^{\mathrm{u}}\right)$ must treat an implication $C \rightarrow D$ with $C \neq A$. Since $\mathbf{G b u}$ has the subformula property, the main formulas of $\rightarrow R_{2}$ applications belong to $\operatorname{Sf}\left(\sigma_{1}\right)$. Thus, $\mathrm{BU}(\mathcal{B})$ is bounded by the number $\# \operatorname{Sf}\left(\sigma_{1}\right)$ of subformulas of $\sigma_{1}$. Since $\# \operatorname{Sf}\left(\sigma_{1}\right) \leq\left|\sigma_{1}\right|$, we get $\mathrm{BU}(\mathcal{B}) \leq\left|\sigma_{1}\right|$.

Since between two ub-pairs of $\mathcal{B}$ a bu-pair must occur, $\mathrm{UB}(\mathcal{B}) \leq \mathrm{BU}(\mathcal{B})+1$; by Lemma $2, \mathrm{UB}(\mathcal{B})$ is finite. We can conclude:

Proposition 1. Every Gbu-branch has finite length.

As a consequence, every $\mathbf{G b u}$-tree has finite depth and $\mathbf{G b u}$ is terminating.

\section{The refutation calculus $\mathrm{Rbu}$}

In this section, following the ideas of $[3,9]$, we introduce the refutation calculus Rbu for deriving intuitionistic unprovability. Intuitively, an Rbu-derivation $\pi$ of a sequent $\sigma^{\mathrm{u}}$ is a sort of "constructive proof" of refutability of $\sigma^{\mathrm{u}}$ in the sense that from $\pi$ we can extract a countermodel $\operatorname{Mod}(\pi)$ for $\sigma^{\mathrm{u}}$.

We denote with $\Gamma^{\text {At }}$ a finite set of propositional variables and with $\Gamma^{\rightarrow}$ a finite set of implicative formulas. A sequent $\sigma$ is irreducible iff $\sigma=\left[\Gamma^{\rightarrow}, \Gamma^{\mathrm{At}} \stackrel{l}{\Rightarrow} H\right]$ 
with $H \in\{\perp\} \cup\left(\mathcal{V} \backslash \Gamma^{\mathrm{At}}\right)$ and $\left(l=\mathrm{b}\right.$ or $\left.\Gamma^{\rightarrow}=\emptyset\right)$. The rules of $\mathbf{R b u}$ are given in Fig. 3. As in $\mathbf{G b u}$, writing $C, \Gamma$ in the conclusion of a rule, we assume that $C \notin \Gamma$. The notions of $\mathbf{R b u}$-tree, $\mathbf{R b u}$-derivation and $\mathbf{R b u}$-branch are defined analogously to those for $\mathbf{G b u}$.

The rule $\mathrm{S}_{u}^{\text {At }}$ has a premise $\left[\Gamma^{\rightarrow}, \Gamma^{\mathrm{At}} \stackrel{\mathrm{b}}{\Rightarrow} A\right]$ for every $A$ such that $A \rightarrow B \in$ $\Gamma^{\rightarrow}$; since $\Gamma^{\rightarrow} \neq \emptyset$, there exists at least one premise. The rule $\mathrm{S}_{u}^{\vee}$ is similar and has at least two premises. All the premises of $S_{u}^{A t}$ and $S_{u}^{\vee}$ are b-sequents.

It is easy to check that an $\mathbf{R b u}$-branch is also a $\mathbf{G b u}$-branch ${ }^{2}$. Accordingly, Proposition 1 implies that the calculus $\mathbf{R b u}$ is terminating. In the following we prove that $\mathbf{R b u}$ is sound in the following sense:

Theorem 2 (Soundness of $\mathbf{R b u}$ ). If an $\mathrm{u}$-sequent $\sigma^{\mathrm{u}}$ is provable in $\mathbf{R b u}$, then $\sigma^{\mathrm{u}}$ is refutable.

Example 2. Let $S=((\neg \neg p \rightarrow p) \rightarrow(\neg p \vee p)) \rightarrow(\neg \neg p \vee \neg p)$ be an instance of the Scott principle [1], where $\neg Z=Z \rightarrow \perp$. We show the Rbu-derivation $\pi_{1}$ of $\stackrel{\stackrel{u}{\Rightarrow}}{\Rightarrow} S$ ].

$$
S=A \rightarrow(\neg \neg p \vee \neg p) \quad A=(\neg \neg p \rightarrow p) \rightarrow(\neg p \vee p)
$$

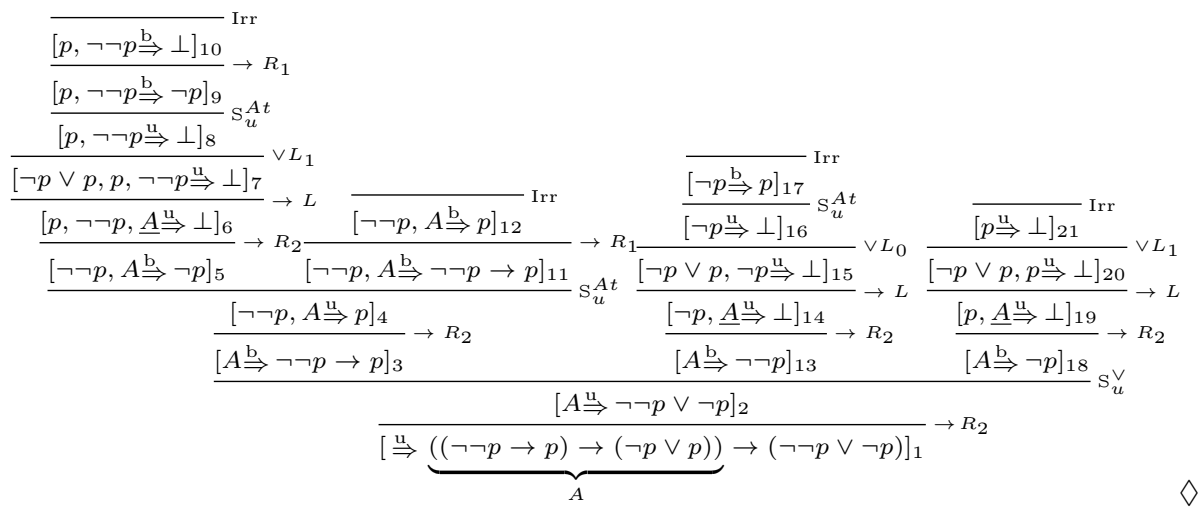

Soundness of Rbu Let $\pi$ be an Rbu-derivation with root $\sigma^{\mathrm{b}}=\left[\Gamma^{\rightarrow}, \Gamma^{\mathrm{At}} \stackrel{\mathrm{b}}{\Rightarrow} H\right]$. By $\Pi\left(\pi, \sigma^{\mathrm{b}}\right)$ we denote the maximal subtree of $\pi$ having root $\sigma^{\mathrm{b}}$ and only containing b-sequents (that is, any subtree of $\pi$ with root $\sigma^{\mathrm{b}}$ extending $\Pi\left(\pi, \sigma^{\mathrm{b}}\right)$ contains at least one u-sequent). Since only the rules $\wedge R_{k}, \vee R$ and $\rightarrow R_{1}$ can be applied in $\Pi\left(\pi, \sigma^{\mathrm{b}}\right)$, every leaf $\sigma^{\prime}$ of $\Pi\left(\pi, \sigma^{\mathrm{b}}\right)$ has the form $\left[\Gamma^{\rightarrow}, \Gamma^{\mathrm{At}} \stackrel{\mathrm{b}}{\Rightarrow} H^{\prime}\right]$, where $H^{\prime} \in \operatorname{Sf}(H)$; moreover, $\sigma^{\prime}$ is either an irreducible sequent (hence a leaf of $\pi$ ) or the conclusion of an application of $\rightarrow R_{2}$ (the only rule of $\mathbf{R b u}$ which, read bottom-up, "unblocks" a b-sequent). Thus, $\pi$ can be displayed as in Fig. 4 . The sequents $\sigma_{1}^{\mathrm{u}}, \ldots, \sigma_{n}^{\mathrm{u}}(n \geq 0)$ are called the u-successors of $\sigma^{\mathrm{b}}$ in $\pi$, while the sequents $\tau_{1}^{\mathrm{b}}, \ldots, \tau_{m}^{\mathrm{b}}(m \geq 0)$ are the $i$-successors (irreducible successors) of $\sigma^{\mathrm{b}}$ in $\pi$. Let $\mathrm{d}(\pi)$ be the depth of $\pi$; if $\mathrm{d}(\pi)=0$, then $\sigma^{\mathrm{b}}$ coincides with $\tau_{1}^{\mathrm{b}}$, hence $\sigma^{\mathrm{b}}$ has no u-successors and has itself as only i-successor.

${ }^{2}$ The converse in general does not hold since the rule $\vee R$ of $\mathbf{R b u}$ requires a b-sequent as conclusion. 


$$
\begin{aligned}
& \vdots \pi_{i} \\
& \frac{\sigma_{i}^{\mathrm{u}}=\left[\Gamma^{\rightarrow}, \Gamma^{\mathrm{At}}, A_{i} \stackrel{\mathrm{u}}{\Rightarrow} B_{i}\right]}{\ldots} \rightarrow R_{i}^{\mathrm{b}=\left[\Gamma^{\rightarrow}, \Gamma^{\mathrm{At}} \stackrel{\mathrm{b}}{\Rightarrow} A_{i} \rightarrow B_{i}\right]} \rightarrow R_{2} \quad \ldots \quad \overline{\tau_{j}^{\mathrm{b}}=\left[\Gamma^{\rightarrow}, \Gamma^{\mathrm{At}} \stackrel{\mathrm{b}}{\Rightarrow} H_{j}\right]} \operatorname{Irr} \quad \ldots \\
& \vdots \Pi\left(\pi, \sigma^{\mathrm{b}}\right) \\
& \sigma^{\mathrm{b}}=\left[\Gamma^{\rightarrow}, \Gamma^{\mathrm{At}} \stackrel{\mathrm{b}}{\Rightarrow} H\right]
\end{aligned}
$$

Fig. 4. Structure of an Rbu-derivation $\pi$ of $\sigma^{\mathrm{b}}=\left[\Gamma^{\rightarrow}, \Gamma^{\mathrm{At}} \stackrel{\mathrm{b}}{\Rightarrow} H\right]$.

Now, let us consider an Rbu-derivation $\pi$ of an u-sequent $\sigma^{\mathrm{u}}$ having root rule $\mathcal{R}=\mathrm{S}_{u}^{\text {At }}$ or $\mathcal{R}=\mathrm{S}_{u}^{\vee}$. Every premise $\sigma^{\prime}$ of $\mathcal{R}$ is a b-sequent and the subderivation of $\pi$ with root $\sigma^{\prime}$ has the structure shown in Fig. 4 . The set of the u-successors of $\sigma^{\mathrm{u}}$ in $\pi$ is the union of the sets of u-successors in $\pi$ of the premises of $\mathcal{R}$; the set of the i-successors of $\sigma^{\mathrm{u}}$ in $\pi$ is defined analogously. To display a proof $\pi$ of this kind we use the concise notation of Fig. 5 .

Example 3. Let us consider the Rbu-derivation $\pi_{1}$ in Ex. 2. The u-successors and i-successors are defined as follows:

\begin{tabular}{|c|c|c|}
\hline u-sequent & u-successors & i-successors \\
\hline$\sigma_{2}$ & $\sigma_{4}, \sigma_{14}, \sigma_{19}$ & \\
\hline$\sigma_{4}$ & $\sigma_{6}$ & $\sigma_{12}$ \\
\hline$\sigma_{8}$ & & $\sigma_{10}$ \\
\hline$\sigma_{16}$ & & $\sigma_{17}$ \\
\hline
\end{tabular}

Now we describe how to extract from an Rbu-derivation of an u-sequent $\sigma^{\mathrm{u}}$ a Kripke countermodel $\operatorname{Mod}(\pi)$ for $\sigma^{\mathrm{u}} \cdot \operatorname{Mod}(\pi)$ is defined by induction on $\mathrm{d}(\pi)$. By $\mathcal{K}^{1}\left(\rho, \Gamma^{\text {At }}\right)$ we denote the Kripke model $\mathcal{K}=\langle\{\rho\},\{(\rho, \rho)\}, \rho, V\rangle$ consisting of only one world $\rho$ such that $V(\rho)=\Gamma^{\text {At }}$. Let $\mathcal{R}$ be the root rule of $\pi$.

(K1) If $\mathcal{R}=$ Irr, then $\mathrm{d}(\pi)=0$ and $\sigma^{\mathrm{u}}=\left[\Gamma^{\mathrm{At}} \stackrel{\mathrm{u}}{\Rightarrow} H\right]$ (being $\sigma^{\mathrm{u}}$ irreducible, $\left.\Gamma^{\rightarrow}=\emptyset\right)$. We set $\operatorname{Mod}(\pi)=\mathcal{K}^{1}\left(\rho, \Gamma^{\mathrm{At}}\right)$, with $\rho$ any element.

(K2) Let $\mathcal{R}$ be different from Irr, $\mathrm{S}_{u}^{\mathrm{At}}, \mathrm{S}_{u}^{\vee}$ and let $\pi^{\prime}$ be the only immediate subderivation of $\pi$. Then, $\operatorname{Mod}(\pi)=\operatorname{Mod}\left(\pi^{\prime}\right)$.

(K3) Let $\mathcal{R}$ be $\mathrm{S}_{u}^{\mathrm{At}}$ or $\mathrm{S}_{u}^{\vee}$ and let $\pi$ be displayed as in Fig. 5 .

If $n=0$, then $\mathcal{K}$ is the model $\mathcal{K}^{1}\left(\rho, \Gamma^{\mathrm{At}}\right)$, with $\rho$ any element.

Let $n>0$ and, for every $i \in\{1, \ldots, n\}$, let $\operatorname{Mod}\left(\pi_{i}\right)=\left\langle P_{i}, \leq_{i}, \rho_{i}, V_{i}\right\rangle$. Without loss of generality, we can assume that the $P_{i}$ 's are pairwise disjoint. Let $\rho$ be an element not in $\bigcup_{i \in\{1, \ldots, n\}} P_{i}$ and let $\mathcal{K}=\langle P, \leq, \rho, V\rangle$ be the model such that:

$-P=\{\rho\} \cup \bigcup_{i \in\{1, \ldots, n\}} P_{i}$; 

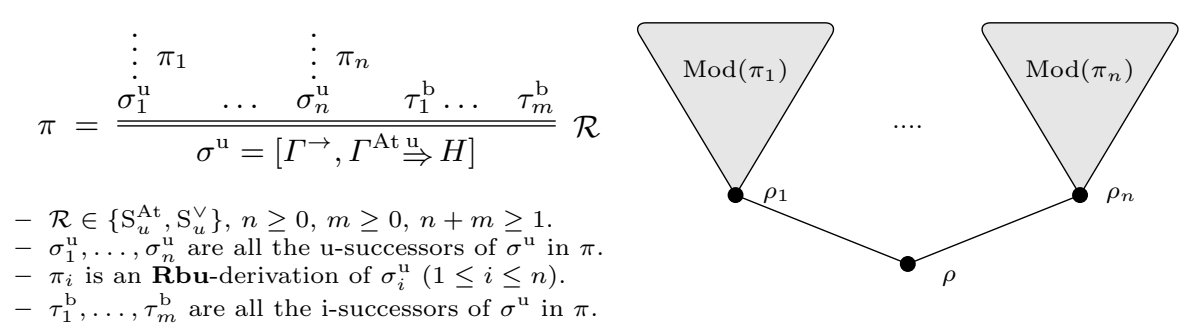

Fig. 5. An Rbu-derivation $\pi$ with root rule $\mathrm{S}_{u}^{\mathrm{At}}$ or $\mathrm{S}_{u}^{\vee}$ and the $\operatorname{model} \operatorname{Mod}(\pi)$.

$-\leq=\{(\rho, \alpha) \mid \alpha \in P\} \cup \bigcup_{i \in\{1, \ldots, n\}} \leq_{i}$

- $V(\rho)=\Gamma^{\text {At }}$ and, for every $i \in\{1, \ldots, n\}$ and $\alpha \in P_{i}, V(\alpha)=V_{i}(\alpha)$.

Then $\operatorname{Mod}(\pi)=\mathcal{K}$. The model $\operatorname{Mod}(\pi)$ is represented in Fig. 5 .

Example 4. We show the Kripke model $\operatorname{Mod}\left(\pi_{1}\right)$ extracted from the Rbu-derivation $\pi_{1}$ of Ex. 2. The model is displayed as a tree with the convention that $w<w^{\prime}$ if the world $w$ is drawn below $w^{\prime}$. For each $w_{i}$, we list the propositional variables in $V\left(w_{i}\right)$. We inductively define the models $\operatorname{Mod}\left(\pi_{i}\right)$ for every

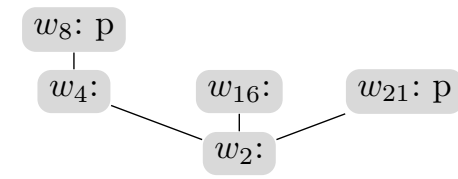
$i$ such that $\sigma_{i}=\left[\Gamma_{i} \stackrel{\mathrm{u}}{\Rightarrow} H_{i}\right]$ is an u-sequent. At each step one can check that $\operatorname{Mod}\left(\pi_{i}\right), \rho_{i} \triangleright \sigma_{i}$, where $\rho_{i}$ is the root of $\operatorname{Mod}\left(\pi_{i}\right)$. Hence, $\operatorname{Mod}\left(\pi_{1}\right), w_{2} \nVdash S$ $\left(\operatorname{Mod}\left(\pi_{1}\right)\right.$ is a countermodel for $\left.S\right)$.

- By Point (K3), since $\sigma_{8}$ has no u-successors (see Ex. 3), $\operatorname{Mod}\left(\pi_{8}\right)=\mathcal{K}^{1}\left(w_{8},\{p\}\right)$. Similarly, $\operatorname{Mod}\left(\pi_{16}\right)=\mathcal{K}^{1}\left(w_{16}, \emptyset\right)$.

- Since $\sigma_{21}$ is irreducible, by Point $(\mathrm{K} 1) \operatorname{Mod}\left(\pi_{21}\right)=\mathcal{K}^{1}\left(w_{21},\{p\}\right)$.

- By Point $(\mathrm{K} 2), \operatorname{Mod}\left(\pi_{6}\right)=\operatorname{Mod}\left(\pi_{7}\right)=\operatorname{Mod}\left(\pi_{8}\right)$. Similarly,

$\operatorname{Mod}\left(\pi_{14}\right)=\operatorname{Mod}\left(\pi_{15}\right)=\operatorname{Mod}\left(\pi_{16}\right)$ and $\operatorname{Mod}\left(\pi_{19}\right)=\operatorname{Mod}\left(\pi_{20}\right)=\operatorname{Mod}\left(\pi_{21}\right)$.

- By Point (K3), $\operatorname{Mod}\left(\pi_{4}\right)$ is obtained by extending with $w_{4}$ the $\operatorname{model} \operatorname{Mod}\left(\pi_{6}\right)$ (indeed, $\sigma_{6}$ is the only u-successor of $\left.\sigma_{4}\right)$ and $V\left(w_{4}\right)=\Gamma_{4} \cap \mathcal{V}=\emptyset$. Similarly, $\operatorname{Mod}\left(\pi_{2}\right)$ is obtained by gluing on $w_{2}$ the models generated by the u-successors $\sigma_{4}, \sigma_{14}$ and $\sigma_{19}$ of $\sigma_{2}$ and $V\left(w_{2}\right)=\Gamma_{2} \cap \mathcal{V}=\emptyset$.

- Finally, $\operatorname{Mod}\left(\pi_{1}\right)=\operatorname{Mod}\left(\pi_{2}\right)$ by Point $(\mathrm{K} 2)$.

We prove the soundness of Rbu. Given an Rbu-tree $\pi$ with $\operatorname{root}\left[\Gamma^{\rightarrow}, \Gamma^{\mathrm{At}} \stackrel{\mathrm{b}}{\Rightarrow} H\right]$ and only containing b-sequents, every leaf of $\pi$ has the form $\left[\Gamma^{\rightarrow}, \Gamma^{\mathrm{At}} \stackrel{\mathrm{b}}{\Rightarrow} H^{\prime}\right]$.

Lemma 3. Let $\pi$ be an Rbu-tree with root $\sigma^{\mathrm{b}}=\left[\Gamma^{\rightarrow}, \Gamma^{\mathrm{At}} \stackrel{\mathrm{b}}{\Rightarrow} H\right]$ and only containing b-sequents, let $\sigma_{1}^{\mathrm{b}}=\left[\Gamma^{\rightarrow}, \Gamma^{\mathrm{At}} \stackrel{\mathrm{b}}{\Rightarrow} H_{1}\right], \ldots, \sigma_{n}^{\mathrm{b}}=\left[\Gamma^{\rightarrow}, \Gamma^{\mathrm{At}} \stackrel{\mathrm{b}}{\Rightarrow} H_{n}\right]$ be the leaves of $\pi$. Let $\mathcal{K}=\langle P, \leq, \rho, V\rangle$ be a Kripke model and $\alpha \in P$ such that:

(H1) $\mathcal{K}, \alpha \nVdash H_{i}$, for every $i \in\{1, \ldots, n\}$;

(H2) $\mathcal{K}, \alpha \Vdash Z$, for every $Z \in \Gamma^{\rightarrow} \cap \operatorname{Sf}(H)$; 
(H3) $V(\alpha)=\Gamma^{\mathrm{At}}$.

Then, $\mathcal{K}, \alpha \nVdash H$.

Proof. By induction on $\mathrm{d}(\pi)$. If $\mathrm{d}(\pi)=0$, then $\sigma^{\mathrm{b}}=\sigma_{1}^{\mathrm{b}}$ and the assertion immediately follows by (H1). Let us assume that $\mathrm{d}(\pi)>0$ and let $\mathcal{R}$ be the root rule of $\pi$. Since both the conclusion and the premises of $\mathcal{R}$ are b-sequents, $\mathcal{R}$ is one of the rules $\wedge R_{k}, \vee R$ and $\rightarrow R_{1}$. The proof proceeds by cases on $\mathcal{R}$. The cases $\mathcal{R} \in\left\{\wedge R_{k}, \vee R\right\}$ immediately follow by the induction hypothesis.

If $\mathcal{R}$ is $\rightarrow R_{1}$, then $\sigma^{\mathrm{b}}=\left[\Gamma^{\rightarrow}, \Gamma^{\mathrm{At}} \stackrel{\mathrm{b}}{\Rightarrow} A \rightarrow B\right]$, the premise of $\mathcal{R}$ is $\sigma^{\prime}=$ $\left[\Gamma^{\rightarrow}, \Gamma^{\mathrm{At}} \stackrel{\mathrm{b}}{\Rightarrow} B\right]$ and, by the side condition, $\Gamma^{\rightarrow}, \Gamma^{\mathrm{At}} \vdash_{\mathcal{E}} A$. By induction hypothesis on the subderivation of $\pi$ having root $\sigma^{\prime}$, we get $\mathcal{K}, \alpha \nVdash B$. We show that $\mathcal{K}, \alpha \Vdash A$. Let $\Gamma_{A}=\left(\Gamma^{\rightarrow} \cap \operatorname{Sf}(A)\right) \cup \Gamma^{\mathrm{At}}$. Since $\Gamma_{A} \cap \operatorname{Sf}(A)=\left(\Gamma^{\rightarrow} \cup \Gamma^{\mathrm{At}}\right) \cap \operatorname{Sf}(A)$ and $\Gamma^{\rightarrow}, \Gamma^{\mathrm{At}} \vdash_{\mathcal{E}} A$, by $(\mathcal{E} 1)$ we get $\Gamma_{A} \vdash_{\mathcal{E}} A$. By the hypothesis (H2) and (H3) of the lemma, it holds that $\mathcal{K}, \alpha \Vdash \Gamma_{A}$; by $(\mathcal{E} 6)$, we deduce $\mathcal{K}, \alpha \Vdash A$. Thus $\mathcal{K}, \alpha \Vdash A$ and $\mathcal{K}, \alpha \nVdash B$, which implies $\mathcal{K}, \alpha \nVdash A \rightarrow B$.

Now, we show that the model $\operatorname{Mod}(\pi)$ is a countermodel for $\sigma^{\mathrm{u}}$.

Theorem 3. Let $\pi$ be an $\mathbf{R b u}$-derivation of an $\mathrm{u}$-sequent $\sigma^{\mathrm{u}}$ and let $\rho$ be the root of $\operatorname{Mod}(\pi)$. Then $\operatorname{Mod}(\pi), \rho \triangleright \sigma^{\mathrm{u}}$.

Proof. By induction on $\mathrm{d}(\pi)$. If $\mathrm{d}(\pi)=0$, then $\operatorname{Mod}(\pi)$ is defined as in (K1) and the assertion immediately follows.

Let $\mathrm{d}(\pi)>0$ and let $\mathcal{R}$ be the root rule of $\pi$. If $\mathcal{R} \notin\left\{\mathrm{S}_{u}^{\mathrm{At}}, \mathrm{S}_{u}^{\vee}\right\}$, the assertion immediately follows by induction hypothesis (the case $\mathcal{R}=\rightarrow R_{1}$ requires (E6)).

Let $\mathcal{R}=\mathrm{S}_{u}^{\vee}$ (the case $\mathcal{R}=\mathrm{S}_{u}^{\mathrm{At}}$ is similar). Let $\sigma^{\mathrm{u}}=\left[\Gamma^{\rightarrow}, \Gamma^{\mathrm{At}} \stackrel{\mathrm{u}}{\Rightarrow} H_{0} \vee H_{1}\right]$ and let $\mathcal{K}=\langle P, \leq, \rho, V\rangle$ be the model $\operatorname{Mod}(\pi)$. By a secondary induction hypothesis on the structure of formulas, we prove that:

(B1) $\mathcal{K}, \rho \nVdash A$, for every $A \rightarrow B \in \Gamma^{\rightarrow}$;

(B2) $\mathcal{K}, \rho \Vdash A \rightarrow B$, for every $A \rightarrow B \in \Gamma^{\rightarrow}$;

(B3) $\mathcal{K}, \rho \nVdash H_{0}$ and $\mathcal{K}, \rho \nVdash H_{1}$.

To prove Point (B1), let $A \rightarrow B \in \Gamma^{\rightarrow}$. By definition of $\mathrm{S}_{u}^{\vee}$, $\pi$ has an immediate subderivation $\pi_{A}$ of $\sigma_{A}^{\mathrm{b}}=\left[\Gamma^{\rightarrow}, \Gamma^{\mathrm{At}} \stackrel{\mathrm{b}}{\Rightarrow} A\right]$ of the form (see Fig. 4):

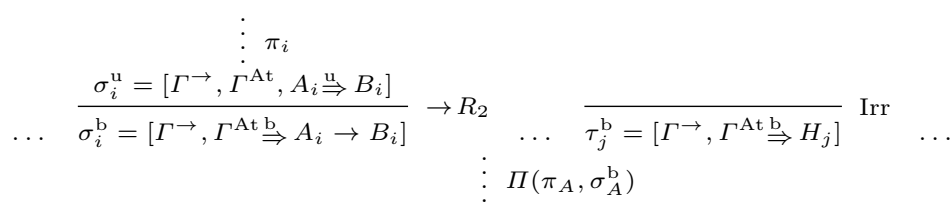

$$
\begin{aligned}
& \sigma_{A}^{\mathrm{b}}=\left[\Gamma^{\rightarrow}, \Gamma^{\mathrm{A}} \stackrel{\mathrm{b}}{\Rightarrow} A\right]
\end{aligned}
$$

We show that $\Pi\left(\pi_{A}, \sigma_{A}^{\mathrm{b}}\right)$ meets the hypothesis (H1)-(H3) of Lemma 3 w.r.t. the root $\rho$ of $\mathcal{K}$, so that we can apply the lemma to infer $\mathcal{K}, \rho \nVdash A$. We prove (H1). Let us assume $n \geq 1$ and let $i \in\{1, \ldots, n\}$; we must show that $\mathcal{K}, \rho \nVdash A_{i} \rightarrow B_{i}$. 
Since $\sigma_{i}^{\mathrm{u}}$ is an u-successor of $\sigma^{\mathrm{u}}$, the root $\rho_{i}$ of $\operatorname{Mod}\left(\pi_{i}\right)$ is an immediate successor of $\rho$ in $\mathcal{K}$. By the main induction hypothesis $\operatorname{Mod}\left(\pi_{i}\right), \rho_{i} \triangleright \sigma_{i}^{\mathrm{u}}$; this implies that $\operatorname{Mod}\left(\pi_{i}\right), \rho_{i} \Vdash A_{i}$ and $\operatorname{Mod}\left(\pi_{i}\right), \rho_{i} \nVdash B_{i}$. Since $\operatorname{Mod}\left(\pi_{i}\right)$ is a submodel of $\mathcal{K}$, we get $\mathcal{K}, \rho_{i} \Vdash A_{i}$ and $\mathcal{K}, \rho_{i} \nVdash B_{i}$, which implies $\mathcal{K}, \rho \nVdash A_{i} \rightarrow B_{i}$. Let $m \geq 1$ and $j \in\{1, \ldots, m\}$. By definition of $\tau_{j}^{\mathrm{b}}$, either $H_{j}=\perp$ or $H_{j} \in \mathcal{V} \backslash \Gamma^{\mathrm{At}}$; in both cases $\mathcal{K}, \rho \nVdash H_{j}$. This proves that hypothesis (H1) of Lemma 3 holds. To prove hypothesis (H2), let $Z \in \Gamma^{\rightarrow} \cap \operatorname{Sf}(A)$. Since $|Z|<|A \rightarrow B|$, by the secondary induction hypothesis on Point (B2) we get $\mathcal{K}, \rho \Vdash Z$. The hypothesis (H3) follows by the definition of $V$ in $\mathcal{K}$. We can apply Lemma 3 to deduce $\mathcal{K}, \rho \nVdash A$, and this proves Point (B1).

We prove Point (B2). Let $\pi$ and $\operatorname{Mod}(\pi)$ be as in Fig. 5 (with $H=H_{0} \vee H_{1}$ ). Let $A \rightarrow B \in \Gamma^{\rightarrow}$ and let $\alpha$ be a world of $\mathcal{K}$ such that $\mathcal{K}, \alpha \Vdash A$; we show that $\mathcal{K}, \alpha \Vdash B$. By Point (B1), $\alpha$ is different from $\rho$. Thus, $n \geq 1$ and, for some $i \in\{1, \ldots, n\}, \alpha$ belongs to $\operatorname{Mod}\left(\pi_{i}\right)$. Let $\rho_{i}$ be the root of $\operatorname{Mod}\left(\pi_{i}\right)$. By the main induction hypothesis, $\operatorname{Mod}\left(\pi_{i}\right), \rho_{i} \triangleright \sigma_{i}^{\mathrm{u}}$; since $A \rightarrow B$ belongs to the left-hand side of $\sigma_{i}^{\mathrm{u}}$, we get $\operatorname{Mod}\left(\pi_{i}\right), \rho_{i} \Vdash A \rightarrow B$, which implies $\mathcal{K}, \rho_{i} \Vdash A \rightarrow B$. Since $\rho_{i} \leq \alpha$ and $\mathcal{K}, \alpha \Vdash A$, we get $\mathcal{K}, \alpha \Vdash B$; thus $\mathcal{K}, \rho \Vdash A \rightarrow B$ and Point (B2) holds.

The proof of Point (B3) is similar to the proof of Point (B1), considering the immediate subderivations of $\pi$ with root sequents $\left[\Gamma^{\rightarrow}, \Gamma^{\mathrm{At}} \stackrel{\mathrm{b}}{\Rightarrow} H_{0}\right]$ and $\left[\Gamma^{\rightarrow}, \Gamma^{\mathrm{At}} \stackrel{\mathrm{b}}{\Rightarrow} H_{1}\right]$. By Points (B2) and (B3) we conclude $\mathcal{K}, \rho \triangleright \sigma^{\mathrm{u}}$.

By Theorem 3, we get the soundness of Rbu stated in Theorem 2 .

\section{The proof-search procedure}

We show that, given an $\mathrm{u}$-sequent $\sigma^{\mathrm{u}}$, either a $\mathbf{G b u}$-derivation or an $\mathbf{R b u}$ derivation of $\sigma^{\mathrm{u}}$ can be built; from this, the completeness of $\mathbf{G b u}$ follows. To this aim, we introduce the function $\mathrm{F}$ of Fig. 6 . A sequent $[\Gamma \stackrel{l}{\Rightarrow} H]$ is in normal form if $l=\mathrm{b}$ implies $\Gamma=\Gamma^{\rightarrow}, \Gamma^{\mathrm{At}}$; given a sequent $\sigma$ in normal form, $\mathrm{F}(\sigma)$ returns either a Gbu-derivation or an Rbu-derivation of $\sigma$. To construct a derivation, we use the auxiliary function $B$ : given a calculus $\mathcal{C} \in\{\mathbf{G b u}, \mathbf{R b u}\}$, a sequent $\sigma$, a set $\mathcal{P}$ of $\mathcal{C}$-trees and a rule $\mathcal{R}$ of $\mathcal{C}, \mathrm{B}(\mathcal{C}, \sigma, \mathcal{P}, \mathcal{R})$ is the $\mathcal{C}$-tree having root sequent $\sigma$, root rule $\mathcal{R}$, and all the $\mathcal{C}$-trees in $\mathcal{P}$ as immediate subtrees.

Proof-search is performed by applying backward the rules of $\mathbf{G b u}$. For instance, the recursive call $\mathrm{F}\left(\left[A, B, \Gamma^{\prime \mathrm{u}} \Rightarrow H\right]\right)$ at line 3 corresponds to the backward application of the rule $\wedge L$ to $\sigma=\left[A \wedge B, \Gamma^{\prime} \stackrel{\mathrm{u}}{\Rightarrow} H\right]$; according to the outcome, at lines 4-5 a Gbu-derivation or an $\mathbf{R b u}$-derivation of $\sigma$ with root rule $\wedge L$ is built. We remark that the input sequent of $\mathrm{F}$ must be in normal form; to guarantee that the recursive invocations are sound, the rules $\vee R_{k}$ and $\rightarrow L$, generating b-sequents, can be backward applied to $[\Gamma \stackrel{\mathrm{u}}{\Rightarrow} H]$ only if $\Gamma$ has the form $\Gamma^{\rightarrow}, \Gamma^{\mathrm{At}}$.

To save space, some instructions are written in a high-level compact form (see, e.g., line 8); the rules used in lines 1 and 32 are defined as follows:

$$
\mathcal{R}_{\mathrm{ax}}([\Gamma \stackrel{l}{\Rightarrow} H])=\left\{\begin{array}{ll}
\perp L & \text { if } \perp \in \Gamma \\
\mathrm{Id} & \text { otherwise }
\end{array} \quad \mathcal{R}_{\mathrm{s}}([\Gamma \stackrel{l}{\Rightarrow} H])= \begin{cases}\vee R & \text { if } l=\mathrm{b} \\
\mathrm{S}_{u}^{\mathrm{At}} & \text { if } l=\mathrm{u} \text { and } H \in \mathcal{V} \\
\mathrm{S}_{u}^{\vee} & \text { otherwise }\end{cases}\right.
$$




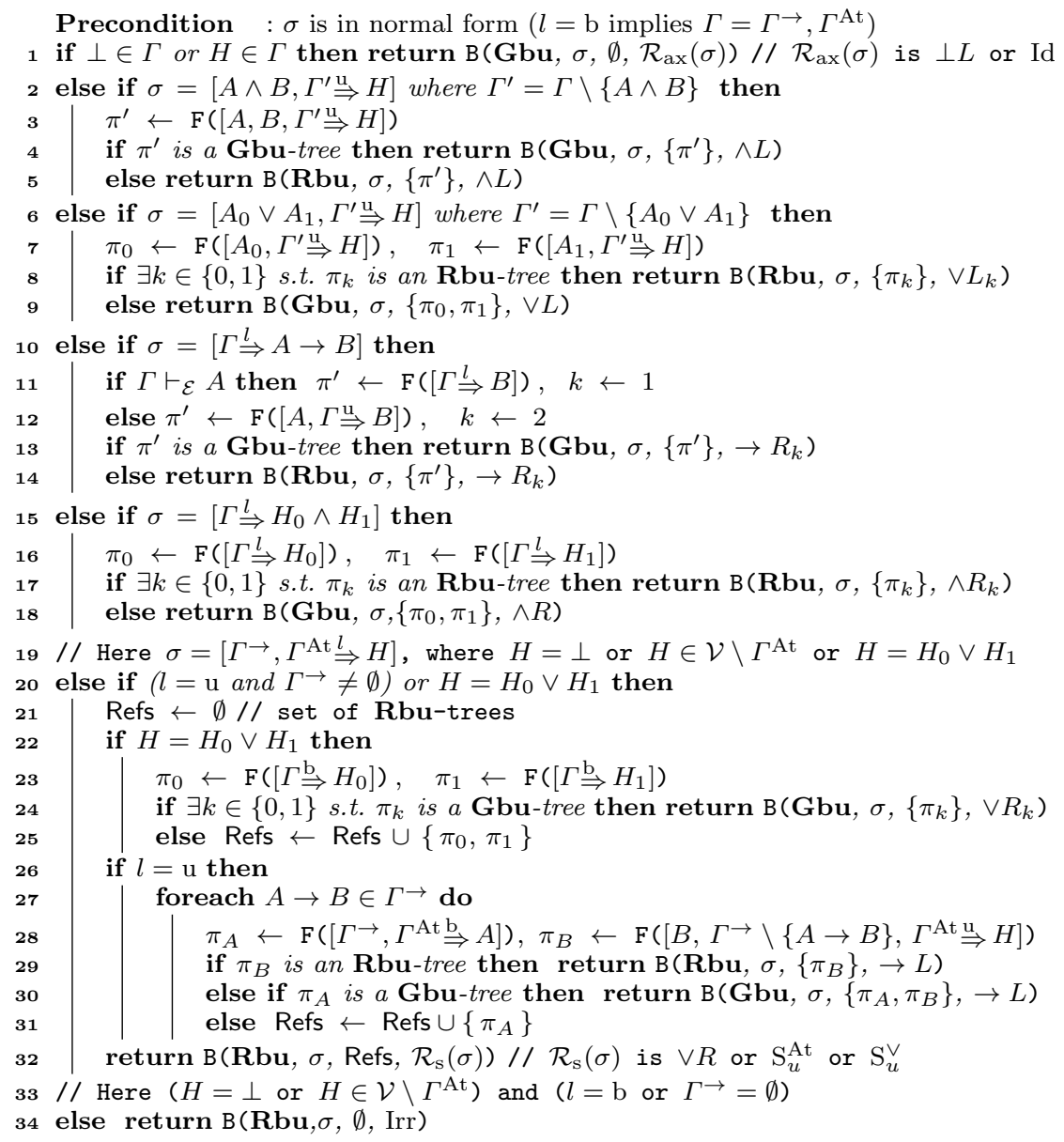

Fig. 6. $\mathrm{F}(\sigma=[\Gamma \stackrel{l}{\Rightarrow} H])$

By $\|\sigma\|$ we denote the maximal length of a $\mathbf{G b u}$-branch starting from $\sigma$ (by Prop. $1,\|\sigma\|$ is finite). Note that, whenever a recursive call $\mathrm{F}\left(\sigma^{\prime}\right)$ occurs along the computation of $\mathrm{F}(\sigma)$, it holds that $\left\|\sigma^{\prime}\right\|<\|\sigma\|$.

In the next lemma we prove the correctness of $F$.

Lemma 4. Let $\sigma$ be a sequent in normal form. Then, $\mathrm{F}(\sigma)$ returns either a Gbu-derivation or an $\mathbf{R b u}$-derivation of $\sigma$.

Proof. By induction on $\|\sigma\|$. If $\|\sigma\|=1, \mathrm{~F}(\sigma)$ does not execute any recursive invocation and the computation ends at line 1 or at line 34 . In the former case, a Gbu-derivation of $\sigma$ is returned. In the latter case, since $\sigma$ is in normal form and none of the conditions at lines 1, 2, 6, 1015,20 holds, the sequent $\sigma$ is irreducible and the tree built at line 34 is an $\mathbf{R b u}$-derivation of $\sigma$. 
Let $\|\sigma\|>1$. Whenever a recursive call $\mathrm{F}\left(\sigma^{\prime}\right)$ occurs, we have that $\left\|\sigma^{\prime}\right\|<$ $\|\sigma\|$ and $\sigma^{\prime}$ is in normal form, hence the induction hypothesis applies to $\mathrm{F}\left(\sigma^{\prime}\right)$. Using this, one can easily show that the arguments of function B are correctly instantiated. We only analyse some cases.

Let us assume that one of the return instructions at lines 8-9 is executed. By induction hypothesis, for every $k \in\{0,1\}, \pi_{k}$ is either a Gbu-proof or an Rbu-derivation of $\sigma_{k}=\left[A_{k}, \Gamma^{\prime} \stackrel{\mathrm{u}}{\Rightarrow} H\right]$. If, for some $k, \pi_{k}$ is an Rbu-derivation of $\sigma_{k}$, then the $\mathbf{R b u}$-tree returned at line 8 is an $\mathbf{R b u}$-derivation of $\sigma$. Otherwise, both $\pi_{0}$ and $\pi_{1}$ are $\mathbf{G b u}$-derivations, hence the value returned at line 9 is a Gbu-derivation of $\sigma$.

Let us assume that $\mathrm{F}(\sigma)$ ends at line 32 ; in this case $\sigma$ satisfies the conditions at lines 19 and 20. If $l=\mathrm{b}$, then $H=H_{0} \vee H_{1}$. Since the condition at line 24 is false, we have Refs $=\left\{\pi_{0}, \pi_{1}\right\}$ and, by induction hypothesis, both $\pi_{0}$ and $\pi_{1}$ are Rbu-derivations. Accordingly, the value returned at line 32 is an Rbu-derivation of $\sigma$ with root rule $\mathcal{R}_{\mathrm{s}}(\sigma)=\vee R$. Let $l=\mathrm{u}$ and let us assume that $H=\perp$ or $H \in \mathcal{V} \backslash \Gamma$. In this case $\sigma=\left[\Gamma^{\rightarrow}, \Gamma^{\mathrm{At}} \stackrel{\mathrm{u}}{\Rightarrow} H\right]$ and the set Refs contains an Rbu-tree $\pi_{A}$ of $\sigma_{A}=\left[\Gamma^{\rightarrow}, \Gamma^{\mathrm{At}} \stackrel{\mathrm{b}}{\Rightarrow} A\right]$ for every $A \rightarrow B \in \Gamma^{\rightarrow}$. By induction hypothesis, $\pi_{A}$ is an $\mathbf{R b u}$-derivation of $\sigma_{A}$, hence line 32 returns an $\mathbf{R b u}$-derivation of $\sigma$ with root rule $\mathcal{R}_{\mathrm{s}}(\sigma)=\mathrm{S}_{u}^{\text {At }}$. The subcase $\left(l=\mathrm{u}\right.$ and $\left.H=H_{0} \vee H_{1}\right)$ is similar.

Finally, we get the completeness of Gbu:

Theorem 4. An u-sequent $\sigma^{\mathrm{u}}$ is provable in $\mathbf{G b u}$ iff $\sigma^{\mathrm{u}}$ is not refutable.

Proof. The $\Rightarrow$-statement follows by the soundness of Gbu. Conversely, let $\sigma^{\mathrm{u}}$ be not refutable. Then, there is no Rbu-derivation $\pi$ of $\sigma^{\mathrm{u}}$; otherwise, by Theorem 3 , from $\pi$ we could extract a countermodel for $\sigma^{\mathrm{u}}$. Since $\sigma^{\mathrm{u}}$ is in normal form, by Lemma 4 the call $\mathrm{F}\left(\sigma^{\mathrm{u}}\right)$ returns a $\mathbf{G b u}$-derivation of $\sigma^{\mathrm{u}}$.

\section{Conclusions and future works}

We have presented Gbu, a terminating sequent calculus for intuitionistic propositional logic. $\mathbf{G b u}$ is a notational variant of $\mathbf{G 3} \mathbf{i}$, where sequents are labelled to mark the right-focused phase. Note that focusing techniques reduce the search space limiting the use of contraction, but they do not guarantee termination of proof-search (see, e.g., the right-focused calculus $L J Q[2]$ ). To get this, one has to introduce extra machinery. An efficient solution is loop-checking implemented by history mechanisms [6,7]. Here we propose a different approach, based on an evaluation relation defined on sequents. Histories require space to store the right formulas already used so to direct and possibly stop the proof-search. Instead, we have to compute evaluation relations when right-implication is treated. We remark that, with an appropriate implementation of the involved data structures (see [4]), the evaluation relation $\vdash_{\tilde{\mathcal{E}}}$ defined in Section 2 can be computed in time linear in the size of the arguments. Hence, we get by means of computation what history mechanisms get using memory. Although a strict comparison 
is hard, to stress the difference between the two approaches we provide an example where $\mathbf{G b u}$ outperforms history-based calculi. Let $\sigma=\left[\Gamma^{\rightarrow} \stackrel{\mathrm{u}}{\Rightarrow} \perp\right]$, where $\Gamma^{\rightarrow}=\left\{p_{1} \rightarrow \perp, \ldots, p_{n} \rightarrow \perp\right\}$ and the $p_{i}$ 's are distinct propositional variables. The only rule that can be used to derive $\sigma$ is $\rightarrow L$. For every $p_{i} \rightarrow \perp$ chosen as main formula, the right-hand premise is provable in $\mathbf{G b u}$, while the left-hand premise $\sigma_{i}^{\mathrm{b}}=\left[\Gamma^{\rightarrow} \stackrel{\mathrm{b}}{\Rightarrow} p_{i}\right]$ is not. Thus, we have a backtrack point which forces the application of $\rightarrow L$ in all possible ways. Being $\sigma_{i}^{\mathrm{b}}$ blocked, the unprovability of $\sigma_{i}^{\mathrm{b}}$ is immediately certified. With the calculi in [7], the search process is similar, but to assert the unprovability of $\left[\Gamma^{\rightarrow} \Rightarrow p_{i}\right]$ one has to chain up to $n$ applications of $\rightarrow L$ and build an history set containing all the $p_{i}$ 's.

Differently from the history mechanisms, Gbu only exploits the information in the left-hand side of a sequent. We are investigating the use of more expressive evaluation relations to better grasp the information conveyed by a sequent and further reduce the search space. Finally, we aim to extend the use of these techniques to other logics having a Kripke semantics.

\section{References}

1. A. Chagrov and M. Zakharyaschev. Modal Logic. Oxford University Press, 1997.

2. R. Dyckhoff and S. Lengrand. LJQ: A Strongly Focused Calculus for Intuitionistic Logic. In A. Beckmann et al., editor, CiE, volume 3988 of LNCS, pages 173-185. Springer, 2006.

3. M. Ferrari, C. Fiorentini, and G. Fiorino. Contraction-free linear depth sequent calculi for intuitionistic propositional logic with the subformula property and minimal depth counter-models. Journal of Automated Reasoning, 2012. doi:10.1007/s10817012-9252-7.

4. M. Ferrari, C. Fiorentini, and G. Fiorino. Simplification rules for intuitionistic propositional tableaux. ACM Transactions on Computational Logic (TOCL), 13(2):14:1-14:23, 2012.

5. D.M. Gabbay and N. Olivetti. Goal-Directed Proof Theory. Springer, 2000.

6. A. Heuerding, M. Seyfried, and H. Zimmermann. Efficient loop-check for backward proof search in some non-classical propositional logics. In P. Miglioli et al., editor, TABLEAUX 96, volume 1071 of LNCS, pages 210-225. Springer, 1996.

7. J. M. Howe. Two loop detection mechanisms: A comparision. In D. Galmiche, editor, TABLEAUX 97, volume 1227 of $L N C S$, pages 188-200. Springer, 1997.

8. F. Massacci. Simplification: A general constraint propagation technique for propositional and modal tableaux. In H.C.M. de Swart, editor, TABLEAUX 98, volume 1397 of LNCS, pages 217-231. Springer, 1998.

9. L. Pinto and R. Dyckhoff. Loop-free construction of counter-models for intuitionistic propositional logic. In M. Behara et al., editor, Symposia Gaussiana, Conference A, pages 225-232. Walter de Gruyter, Berlin, 1995.

10. A.S. Troelstra and H. Schwichtenberg. Basic Proof Theory, volume 43 of Cambridge Tracts in Theoretical Computer Science. Cambridge University Press, 1996. 